\title{
La agencia mercantil desde el tratado de libre comercio entre Colombia y Estados Unidos ${ }^{1}$
}

\section{The commercial agency since the free trade agreement between Colombia and the United States}

Recibido: 21 de abril de 2016 - Revisado: 12 de agosto de 2016 - Aceptado: 20 de diciembre de 2016

Juan Sebastián Alejandro Perilla Granados ${ }^{2}$

\section{Resumen}

El contrato de agencia mercantil tiene características específicas que lo diferencian de otros contratos de distribución, las cuales se condensan en elementos esenciales, naturales y accidentales. Aunque tales elementos parecen tener cierta aceptación en las fuentes jurídicas, con la entrada en vigencia del Acuerdo de Promoción Comercial entre la República de Colombia y los Estados Unidos de América (también llamado Tratado de Libre Comercio, TLC) se presenta un posible caso de derogatoria implícita. Para probar esta hipótesis se analiza el rol de los tratados internacionales en el sistema de fuentes del derecho mercantil, para que a través de la teoría del monismo impuro sea posible formular un nuevo alcance del contrato de agencia mercantil.

\section{Palabras clave}

Agencia mercantil, tratado de libre comercio, derogatoria implícita, fuentes del derecho comercial, iusteoría.

\begin{abstract}
The commercial agency contract has specific characteristics that differentiate it from other distribution contracts, which are condensed into essential, natural and accidental elements. Although such elements seem to have some acceptance in legal sources, with the entry into force of the Trade Promotion Agreement between the Republic of Colombia and the United States of America (also called the Free Trade Agreement, FTA) a possible case of implicit repeal is presented. To test this hypothesis, the role of international treaties in the system of sources of commercial law is analyzed, so that through the theory of impure monism it is possible to formulate a new scope of the commercial agency contract.
\end{abstract}

\section{Keywords}

Commercial agency, free trade agreement, implicit repeal, sources of commercial law, iustheory.

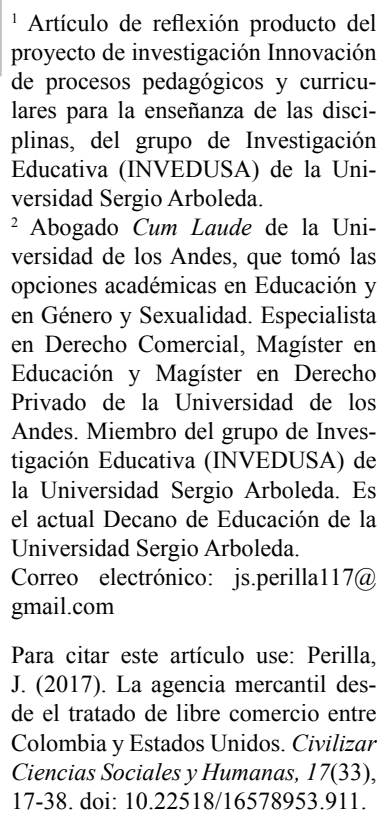

${ }^{1}$ Artículo de reflexión producto del proyecto de investigación Innovación de procesos pedagógicos y curriculares para la enseñanza de las disciplinas, del grupo de Investigación Educativa (INVEDUSA) de la Universidad Sergio Arboleda.

2 Abogado Cum Laude de la Universidad de los Andes, que tomó las opciones académicas en Educación y en Género y Sexualidad. Especialista en Derecho Comercial, Magíster en Educación y Magíster en Derecho Privado de la Universidad de los Andes. Miembro del grupo de Investigación Educativa (INVEDUSA) de la Universidad Sergio Arboleda. Es el actual Decano de Educación de la Universidad Sergio Arboleda.

Correo electrónico: js.perilla117@ gmail.com

Para citar este artículo use: Perilla, J. (2017). La agencia mercantil desde el tratado de libre comercio entre Colombia y Estados Unidos. Civilizar Ciencias Sociales y Humanas, 17(33), 17-38. doi: 10.22518/16578953.911. 


\section{Introducción}

En Colombia, el contrato de agencia mercantil se ha caracterizado desde sus orígenes por responder a principios propios del mercado, según los cuales priman las relaciones de la cotidianidad sobre eventuales solemnidades (Morales, 1988). Esto se debe a que el derecho comercial nace como un derecho de clase especializado, alterno a las dinámicas de regulación del poder central y con la capacidad de trascender fronteras con una fuerza expansiva universalizable (Pinzón, 1985) ${ }^{1}$. Así, en el contrato de agencia mercantil basta con que se configuren presupuestos de hecho mínimos para que se generen consecuencias jurídicas con repercusiones personales y patrimoniales relevantes.

Las consecuencias jurídicas principales se configuran al momento de la terminación del contrato, pues el artículo 1324 del Código de Comercio colombiano exige el pago de un porcentaje del promedio de las utilidades recibidas en los últimos periodos en los cuales se haya configurado la agencia mercantil. Además, el agente tiene derecho a que se le paguen una serie de indemnizaciones equitativas que tendrán en cuenta para la fijación del valor, la extensión, importancia y volumen de los negocios que el agente haya adelantado en desarrollo del contrato. Estas características del contrato han sido desarrolladas por la jurisprudencia de la Corte Suprema de Justicia, a través de ejercicios hermenéuticos permanentes y la doctrina da cuenta de estos debates:

Mucha tinta ha corrido alrededor de los contratos de agencia comercial desde su consagración legal en el Decreto 410 de 1971 que, con algunas reformas, sigue siendo nuestro estatuto mercantil vigente. En efecto, tratadistas, jueces, árbitros y magistrados han invertido tiempo y esfuerzo en tratar de desentrañar si las prestaciones que nuestro Código de Comercio consagró a favor de los agentes a la finalización del contrato son renunciables o no; si las normas que las establecen son disposiciones de orden público o si por el contrario, representan previsiones de contenido puramente patrimonial sin trascendencia en el orden social (Cuberos, 2005, p. 2).

Tal ha sido la relevancia del contrato de agencia mercantil, que se tuvo una consideración especial en el proceso de negociación y aprobación final del Acuerdo de Promoción Comercial entre la República de Colombia y los Estados Unidos de América (en adelante, tratado de libre comercio celebrado entre Colombia y Estados Unidos o TLC). De esta forma, se expusieron compromisos de cambio normativo del contrato de agencia mercantil, los cuales llevan, con base en este estudio, a enunciar la hipótesis según la cual los elementos de la naturaleza del contrato de agencia comercial fueron modificados a través de un proceso de derogatoria tácita generado por el tratado de libre comercio celebrado entre Colombia y Estados Unidos.

Para probar la hipótesis formulada, se adoptará la siguiente estructura del razonamiento: primero, se abordará el contrato de agencia mercantil desde sus elementos esenciales, naturales y accidentales; y segundo, se establecerán las consecuencias del tratado de libre comercio celebrado entre Colombia y Estados Unidos en relación con la agencia mercantil. Para determinar esas consecuencias, se dividirá el análisis, a su vez, así: se retomará la teoría actual del sistema de fuentes del derecho comercial haciendo énfasis especial en los tratados internacionales de comercio; luego se complementará dicha teoría desde el rol de los tratados internacionales en el derecho doméstico; y finalmente, se definirán cuáles son los cambios generados por el derecho internacional en el régimen comercial colombiano referente a la agencia mercantil. El trabajo de investigación, base del presente artículo, utiliza métodos de investigación cualitativa, con un enfoque socio-crítico basado en revisión documental. 


\section{Elementos contractuales de la agencia mercantil}

El contrato de agencia mercantil, como todo negocio jurídico, cuenta con tres elementos contractuales: los esenciales, los naturales y los accidentales (Ospina y Ospina, 1998) ${ }^{2}$. Los elementos esenciales hacen referencia a aquellos que deben configurarse para que exista el contrato, pues si no se cumplen, el negocio jurídico degenera en inexistente o en uno diferente (Pájaro, 2009). Los naturales son aquellos que sin ser esenciales se presumen incluidos en el contrato, salvo pacto en contrario (Hinestrosa, 2015). Y finalmente, los accidentales son aquellos que sólo harán parte del contrato si las partes lo disponen expresamente (Angarita, 2005). En el caso de la agencia mercantil, el artículo 1317 del actual Código de Comercio colombiano la define así:

Por medio del contrato de agencia, un comerciante asume en forma independiente y de manera estable el encargo de promover o explotar negocios en un determinado ramo y dentro de una zona prefijada en el territorio nacional, como representante o agente de un empresario nacional o extranjero o como fabricante o distribuidor de uno o varios productos del mismo. La persona que recibe dicho encargo se denomina genéricamente agente.

De esta definición se puede concluir que el objeto del contrato de agencia comercial es aquel encargo de promover, explotar, fabricar o distribuir bienes o servicios de un determinado empresario. Las partes de este contrato son dos comerciantes, es decir, personas que profesionalmente realizan actos de comercio en los términos del estatuto mercantil (Castro de Cifuentes, 2009). Aunque se determina que el agenciado puede ser nacional o extranjero, no es del todo claro si el agente solo puede ser nacional o si puede ostentar ambas calidades. Al no diferenciar, se asume que el agente puede ser nacional o extranjero (Giraldo, 2012).

Para poder iniciar el cumplimiento del objeto contractual, la agencia mercantil cuenta con los siguientes elementos esenciales:
- Encargo de promover o explotar negocios: aunque en estricto sentido se trata del objeto contractual, la doctrina lo ha entendido de forma unánime como un elemento esencial; "ya se trate de la promoción o de la explotación, este elemento de la esencia del contrato se refiere a que el agenciado debe procurar el crecimiento de los negocios del empresario, o al menos el sostenimiento de los mismos" (Giraldo, 2012, p. 9). Tanto la promoción como la explotación redundan en última instancia en beneficio del agenciado y se trata de una especie de mandato general, pues no es necesario que el agente reciba instrucciones precisas sobre lo que debe hacer. Por el contrario, deberá hacer todo lo que esté a su alcance para conquistar el mercado a favor del agenciado a manera de finalidad económica del negocio jurídico.

Especificando los términos, por promoción se debe entender la gestión que complementa los ejercicios de publicidad y que permiten asegurar una efectividad específica para la conquista del mercado por parte del agente (Bonivento, 1999). Por su parte, la explotación se refiere a un término más abstracto que incluye cualquier tipo de negocio que celebre el agente, sea distribución, fabricación o cualquier otro contrato que represente utilidad para el agenciado (Arrubla, 1997). Se debe considerar que estas exigencias implican la consolidación de varios negocios para conquistar el mercado y no se pueden limitar a uno específico que se agote en el tiempo (Cuberos, 2005) 3; " "no es para uno o varios negocios aislados y ocasionales, sino para un conjunto de negocios por medio de los cuales se persigue conquistar y reconquistar clientela, acreditar una marca, ampliar y mantener un merca- 
do de bienes y servicios del agenciado" (Giraldo, 2012, p. 10).

- Independencia: el agente debe actuar de forma independiente y autónoma respecto del agenciado (Zuleta, 2004); "el agente desarrolla su gestión con independencia y autonomía, en la medida en que no está vinculado con el productor mediante lazos de subordinación o dependencia, ni hace parte de su organización" (Sentencia del 15 de diciembre de 2006). Se debe señalar que la independencia es analizada desde la ausencia de subordinación o estructura organizacional, lo cual significa que entre el agente y el agenciado no se puede presentar un contrato laboral (Giraldo, 2012). En este sentido se ha pronunciado la jurisprudencia:

No sobra reiterar que la independencia y autonomía son uno de los presupuestos necesarios en esta clase de vínculos, lo que conlleva la inexistencia de lazos de subordinación o dependencia entre agente y agenciado, que de presentarse desencadenarían en relaciones laborales que lo alejarían de los acuerdos mercantiles a que corresponde (Sentencia del 10 de septiembre de 2013).

En virtud de tal independencia, se debe considerar que al agente le corresponde asumir los riegos generados por los costos de su operación, por lo cual tiene libertad para contratar el personal que hará parte de su empresa y diseñar los métodos para cumplir el objeto de la agencia mercantil.

Como actúa como un profesional independiente, hace suyos los riesgos por los costos que su operación de promoción le demande. Por lo mismo, tiene libertad para designar sus colaboradores, para diseñar los métodos que considere más convenientes para cumplir la misión asumida (Sentencia diciembre 15 de 2006) ${ }^{4}$.

Sin embargo, tal independencia no significa que el agente está totalmente desvinculado del agenciado, pues la jurisprudencia avala la posibilidad de que el primero reciba instrucciones por parte del segundo, así: "Existe independencia y autonomía del agente, por ser ajeno a la estructura organizacional del empresario, sin que ello impida que éste le imparta ciertas instrucciones para el cumplimiento de la labor encomendada, al tenor del artículo 1321 ibidem" (Sentencia julio 24 de 2012) ${ }^{5}$.

- Estabilidad: se trata de un elemento propio de los contratos de distribución, según el cual se debe garantizar la permanencia en el tiempo de la inversión realizada por el agente y se debe asegurar el tiempo suficiente para que se pueda alcanzar el objeto contractual (Arrubla, 1997); "la labor de promoción o explotación no se puede hacer sin que haya un intervalo de tiempo adecuado que permita iniciar un mercado o penetrar uno ya existente" (Giraldo, 2012, p. 8) ${ }^{6}$.

No obstante, la permanencia dada por la estabilidad no implica la configuración de un término indefinido para el contrato de agencia mercantil (Namén, 1995); hay contratos de agencia que por la naturaleza de su objeto exigen términos definidos en situaciones específicas. Además, es posible que la estabilidad se vea desdibujada en los casos en que se configuren justas causas de terminación contractual (Peña, 2010).

- Actuación por cuenta de otro: el agente debe actuar por cuenta del agenciado (Sanín, 1995; Vallejo, 1999)7.

La actuación del agente es por cuenta ajena, en vista de que el impacto del éxito o fracaso de la encomienda se patentiza primordialmente en los estados financieros del agenciado, mientras que por sus labores de conexión 
aquel recibe una remuneración preestablecida (Sentencia del 10 de septiembre de 2013).

Lo anterior implica que las utilidades que se generan en virtud de la agencia, benefician de forma directa al agenciado, razón por la cual la clientela creada es del agenciado durante la vigencia del contrato y a la terminación del mismo (Giraldo, 2012; Namén, 1995). Pero esto no significa que el agente esté exento de asumir ciertos riesgos, pues también involucra al agente al tratarse de un negocio por naturaleza oneroso; el agente asume riesgos en la infraestructura y estrategia diseñadas para desarrollar el objeto contractual, encontrando una afectación directa en la remuneración que está en función de las ganancias que se obtengan ${ }^{8}$.

- Área de negocio específica y zona geográfica delimitada: el contrato de agencia debe recaer sobre un área específica de negocio, para responder de esta forma a los principios de especialidad del derecho comercial (Bonivento, 1999; Perilla, 2015a). La especificidad debe estar delimitada por el giro ordinario de los negocios del agenciado (Arrubla, 1997; Castro, 2009). Además, esta actividad específica debe ser desarrollada en una zona geográfica delimitada en el territorio nacional, permitiendo que un mismo agenciado tenga varios agentes y cada uno se ubique en un territorio diferente (Giraldo, 2012; Namén, 1995).

Los anteriores elementos se deben presentar de manera indispensable para que exista el contrato de agencia. "Deben aparecer todos para que pueda predicarse válidamente su configuración, ya que la falta de uno o varios de ellos implica necesaria y fatalmente que tal convención no existe o que degenera en otro acuerdo de naturaleza diferente" (Sentencia del 4 de abril de 2008). Sin embargo, se debe considerar que en realidad se trata de un contrato de mandato general con unas particularidades específicas ${ }^{9}$. La real diferencia de la agencia comercial con otros contratos de distribución se presenta en los elementos contractuales naturales, los cuales suscitan especial pugna litigiosa en la mayoría de los casos por su naturaleza económica. Estos elementos, que se presumen contratados sin necesidad de ser pactados expresamente y a falta de pacto en contrario, son:

- Remuneración a favor del agente: se trata de un tema ampliamente debatido por la doctrina, pues no es claro si se trata de un elemento esencial o uno natural. Para solucionar el debate se puede considerar que la agencia mercantil es por naturaleza remunerada, lo cual implica que las partes pueden pactar en contrario. Esto significa que pueden existir contratos de agencia mercantil que las partes determinen como gratuitos (Arrubla, 1997).

Algunos autores se oponen a esta interpretación apelando a argumentos deductivos; Giraldo (2012) afirma que "Si el artículo 1322 manda imperativamente el pago de remuneración en tres casos extraordinarios, con mucha mayor razón el agente tiene derecho a ella en el desarrollo normal del contrato" (p. 21). Sin embargo, el hecho que haya una remuneración en casos extraordinarios no significa que las partes estén en la obligación de acogerla en todos los casos (Escobar, s.f.). Si el legislador no incluye expresamente esta disposición, y otras normas como la jurisprudencia tampoco se refieren a ella a manera de precedente vinculante, no es posible crear a través de interpretaciones, requisitos de la esencia a los cuales están obligadas las partes en todos los casos. Específicamente, no es posible señalar que el contrato de agencia mercantil es inexistente si las partes no incluyen en el mismo una 
remuneración periódica a favor del agente.

- Exclusividad a favor del agente: el artículo 1318 del Código de Comercio señala expresamente que "salvo pacto en contrario", el agenciado debe tener un solo agente en una misma zona para el ramo de actividades determinado (Arrubla, 1997). Pero esto no le impide al agenciado tener varios agentes en el mismo territorio nacional; el requisito es que haya un agente por cada uno de los territorios específicos asignados (Talero, 2010).

- Prestaciones a la terminación del contrato: de acuerdo con el artículo 1324 del Código de Comercio, existen dos posibles prestaciones a la terminación del contrato $^{10}$ : una sería la doceava parte de la comisión recibida en los tres últimos años (por cada uno de vigencia del contrato) o el promedio de lo recibido en un lapso menor; y la otra, una indemnización equitativa para el agente cuando el agenciado revoque o termine el contrato de forma unilateral sin justa causa. Este elemento se incluye a manera de elemento natural, pues la misma norma comercial abre la posibilidad para no pagar estas prestaciones si las partes lo acuerdan de forma bilateral o si las causas de terminación son atribuibles al agente ${ }^{11}$.

Finalmente, los elementos contractuales accidentales del contrato de agencia son mucho más escasos que los calificados como esenciales y naturales y se refieren específicamente al término de duración y a la exclusividad a favor del agenciado (Arrubla, 1997). En primer lugar y pese a que la estabilidad se incluye como un elemento esencial, la misma no determina una respuesta generalizada sobre la duración del contrato (Giraldo, 2012). Por esta razón, dependerá de las partes determinar expresamente si se trata de un término fijo o un término indefinido; a falta de determinación expresa la ley no presume nada a manera de regla general aplicable a cualquier caso.

En segundo lugar, el artículo 1319 del Código de Comercio señala expresamente que en el contrato de agencia comercial "podrá pactarse la prohibición para el agente de promover o explotar, en la misma zona y en el mismo ramo, los negocios de dos o más empresarios competidores". De esta forma, a falta de pacto específico, un mismo agente puede tener varios agenciados, configurándose el requisito de elemento accidental.

A manera de conclusión en la Tabla 1 se presenta un esquema con los elementos contractuales de la agencia comercial, para proceder a analizarlos en el marco del tratado de libre comercio celebrado entre Colombia y Estados Unidos.

\section{Tabla 1. Elementos contractuales} de la agencia mercantil

\begin{tabular}{|c|c|}
\hline \multirow{6}{*}{$\begin{array}{l}\text { ELEMENTOS } \\
\text { ESENCIALES }\end{array}$} & $\begin{array}{l}\text { Encargo de promover y explotar } \\
\text { negocios }\end{array}$ \\
\hline & Independencia del agente \\
\hline & Estabilidad \\
\hline & $\begin{array}{l}\text { Actuación del agente por cuenta del } \\
\text { agenciado }\end{array}$ \\
\hline & Área de negocio específica \\
\hline & Zona geográfica delimitada \\
\hline \multirow{3}{*}{$\begin{array}{l}\text { ELEMENTOS } \\
\text { NATURALES }\end{array}$} & Remuneración a favor del agente \\
\hline & Exclusividad a favor del agente \\
\hline & $\begin{array}{l}\text { Prestaciones a la terminación del } \\
\text { contrato }\end{array}$ \\
\hline \multirow{2}{*}{$\begin{array}{c}\text { ELEMENTOS } \\
\text { ACCIDENTALES }\end{array}$} & Término fijo o indefinido \\
\hline & Exclusividad a favor del agenciado \\
\hline
\end{tabular}

Fuente: elaboración propia. 


\section{Características del Tratado de Libre Comercio celebrado entre Colombia y Estados Unidos}

En el año 2003 iniciaron las negociaciones entre Colombia y Estados Unidos tendientes a aprobar el tratado de libre comercio entre ambos países, en cuyas rondas se analizaron desde los primeros momentos los elementos contractuales de la agencia comercial estudiados anteriormente. Esto se debe a que "la protección otorgada por la ley colombiana al agente mercantil, es la principal preocupación legal de las partes, al poner en marcha un sistema de distribución de bienes o servicios" (Talero, 2010, p. 24). En tal sentido, el proceso de negociación giró en torno a "una eventual modificación legislativa que, dicho sea de paso, parece irse abriendo paso dentro del marco de las negociaciones que se vienen adelantando hacia la suscripción del Tratado de Libre Comercio ("TLC") con los Estados Unidos" (Cuberos, 2005 , p. 16). La mencionada modificación legislativa se fundamenta en el proceso de negociación que aquí se presenta:

El equipo negociador por parte de los Estados Unidos solicitó, a partir de la tercera ronda de negociaciones, la elaboración de ciertas modificaciones al régimen vigente orientadas a (i) eliminar la cesantía comercial; (ii) revisar el cálculo de la indemnización para tener en cuenta dentro de ella la remuneración percibida por el agente; (iii) eliminar la presunción de exclusividad que existe a favor del agente, y (iv) señalar expresamente que la fecha de terminación del contrato es una justa causa para su terminación. Aun cuando en rondas posteriores se han incluido variantes y modificaciones a estas propuestas, en esencia lo que busca el equipo negociador de los Estados Unidos es que no haya injerencia legislativa en los aspectos remuneratorios del contrato y que haya un poco más de flexibilidad en cuanto a la indemnización que podría causarse por una indebida terminación del mismo (Cuberos, 2005, pp. 16-17).

Como consecuencia de las negociaciones, la solicitud de Estados Unidos fue siendo reconsiderada y "como producto del intercambio de información entre los equipos durante las doce primeras rondas de negociación, el equipo norteamericano solo conservó tres de los cinco puntos inicialmente solicitados en relación a la legislación del contrato de agencia comercial" (López-Álvarez, 2011). Estos tres puntos terminantes se refirieron a: primero, la no aplicación de la prestación comercial contenida en primer inciso del artículo 1324 del Código de Comercio; segundo, la no aplicación de la indemnización equitativa señalada en el segundo inciso del artículo 1324, para ser reemplazado por daño emergente y lucro cesante; y tercero, constituir como accidental la exclusividad del agenciado a favor del agente.

Aunque Estados Unidos solicitaba que se hicieran estos cambios legislativos antes de la entrada en vigencia del tratado, Colombia se comprometió a hacerlo seis meses después de haber entrado en vigencia el TLC; dichas exigencias fueron incluidas a manera de compromiso en el tratado internacional de libre comercio aprobado a través de la ley 1143 de 2007. Con base en lo anterior y como tema de análisis, esta investigación se cuestiona sobre si se presenta o no una derogatoria táctica que modifique el régimen jurídico sobre la agencia mercantil en Colombia. Para dar respuesta se analizarán, en primer lugar, los tratados internacionales mercantiles como fuente de derecho internacional; a partir de esto se propondrá una modificación a la pirámide de fuentes del derecho comercial desde la vinculatoriedad de las normas de derecho internacional; finalmente se podrá concluir, a partir de estos elementos, si la agencia mercantil cambió parcial e implícitamente sus elementos contractuales desde las normas internacionales a las cuales se somete Colombia.

\section{Tratados internacionales mercantiles como fuente del derecho comercial.}

El sistema jurídico colombiano actual se encuentra inscrito en una iusteoría antiformalista 
(López-Medina, 2004), según la cual el derecho cuenta con una naturaleza abierta y debe ser complementado por múltiples fuentes a través de interpretaciones auténticas (Perilla, 2013) ${ }^{12}$. Esta nueva concepción del derecho establecida por la Constitución Política colombiana de 1991, exige un diferente rol por parte de los operadores jurídicos en relación con las normas y la integración de las mismas en el sistema jurídico; las normas no provienen de manera exclusiva del legislador tradicional sino que pueden ser producidas por otras fuentes como el derecho internacional.

Entre los regímenes jurídicos vigentes actualmente en Colombia, el derecho comer- cial materializa de forma directa la iusteoría del antiformalismo ${ }^{13}$; "el régimen de fuentes del derecho comercial responde de manera directa a las características de la iusteoría antiformalista, lo cual se evidencia al analizar la forma de aplicación de las normas jurídicas comerciales a situaciones mercantiles" (Peri1la, 2015a, p. 20). De esta forma, el derecho comercial se entiende como un entramado de normas abierto que puede ser complementado por múltiples fuentes a través de interpretaciones auténticas. En atención a tal naturaleza antiformalista, Perilla (2015a) formula la siguiente estructura para las fuentes del derecho comercial $^{14}$.

\section{Gráfico 1. Jerarquía de normas comerciales: régimen jurídico comercial actual}

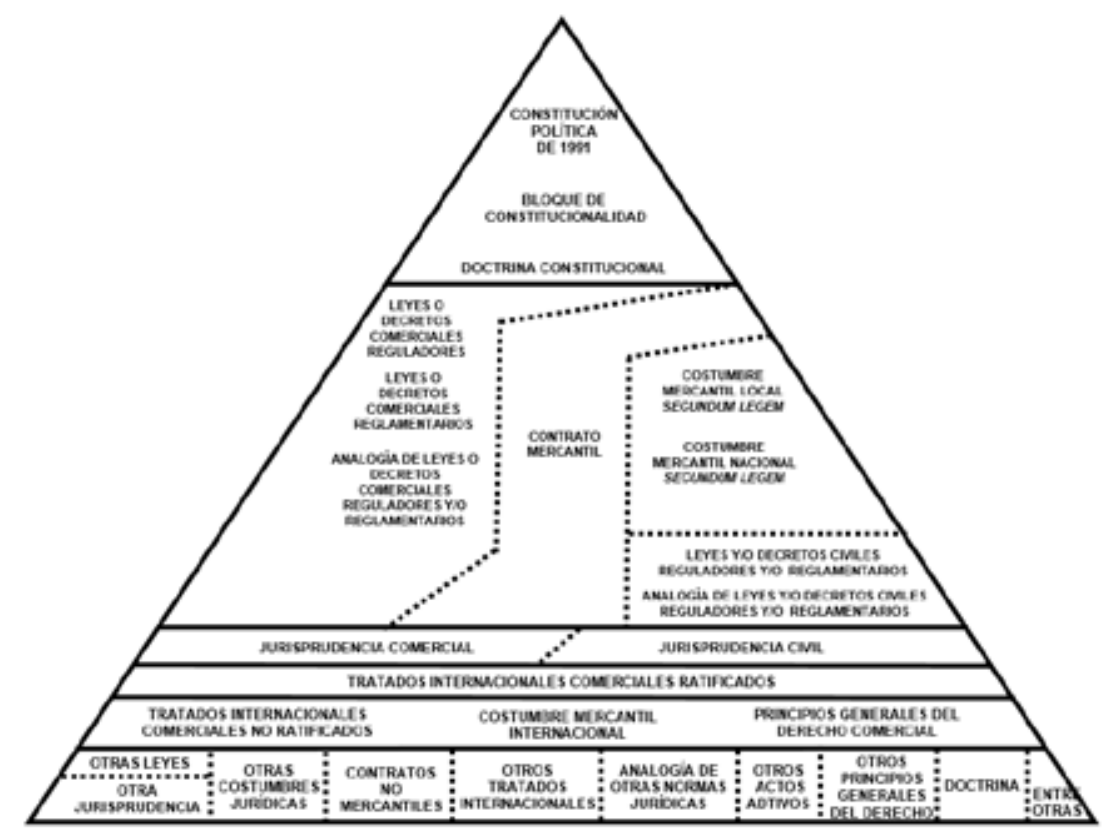

Fuente: elaboración propia con base en Perilla, 2015a.

Se evidencia de esta manera que las normas comerciales internacionales se encuentran vinculadas a la pirámide de fuentes del derecho comercial siguiendo los postulados sobre monismo de Wenzel (1929). Así, los tratados internacionales comerciales ratificados se ubican dentro de la pirámide pero subordinados a las normas domésticas (Azuero, 2009; Benvenisti, 2008). Pese a que se trata de un avance, no se responde en estricto sentido al antiformalismo puesto que con la mencionada subordinación se sigue entendiendo al legislador tradicional como la fuente por excelencia del derecho (Bogdandy, 2008) y las demás normas están llamadas a supeditarse a su voluntad (Brownlie, 2008; Butler, 1985). Con el ánimo de asegurar alineación iusteórica de los tratados internacionales sobre comercio ratificados por Colombia y la iusteoría antiformalista, a 
continuación se traza un ajuste teórico a la pirámide de fuentes de derecho comercial planteada por Perilla (2015a).

\section{Ajuste teórico a la pirámide de fuentes desde los tratados interna- cionales mercantiles.}

En lo referente a sus fuentes, el Código de Comercio colombiano no habla expresamente de los tratados internacionales comerciales ratificados por el Estado (Castro, 2009). Solamente se refiere a los tratados mercantiles de derecho internacional que no están ratificados, ubicándolos al nivel de la costumbre mercantil internacional y de los principios generales del derecho (López-Guzmán, 2007). Esto ha generado múltiples interpretaciones que provienen desde la teoría del monismo, sin reconocer la novedad jurídica presente en Colombia: el monismo impuro (Perilla, 2016) ${ }^{15}$.

Por regla general, el monismo indica que las normas internacionales hacen parte íntegra de la pirámide de fuentes del derecho de cada
Estado y se encuentran vinculadas al derecho interno de dos formas: en un lugar superior a las normas domésticas (Kelsen, 1952; 2009) o en un lugar inferior que ellas (Wenzel, 1920). Se trata de teorías jurídicas nacidas en Europa e introducidas al contexto colombiano a través de lecturas propias en sitios de recepción (Bonilla, 2009; López-Medina, 2004) $)^{16}$. Como consecuencia de estos trasplantes, en Colombia parece existir un proceso de transformación de teorías según el cual los sitios de recepción generan nuevas teorías por medio de misreading (Perilla, 2013; 2015a) ${ }^{17}$. La nueva teoría sugiere que las normas de derecho internacional una vez aprobadas, hacen parte íntegra de las normas domésticas del mismo nivel, es decir, las normas nacionales e internacionales son una sola cuando han sido aprobadas (Ureña, 2012; 2015). En el caso de los tratados internacionales mercantiles, al ser aprobados por leyes del Congreso de la República, se puede afirmar que hacen parte íntegra de la ley comercial y se ubican al nivel del contrato y de la costumbre mercantil (Ureña, 2008). Se trata de una nueva interpretación que modifica en parte la propuesta planteada por Perilla (2015a), a saber:

\section{Gráfico 2. Jerarquía de normas comerciales desde el monismo impuro}

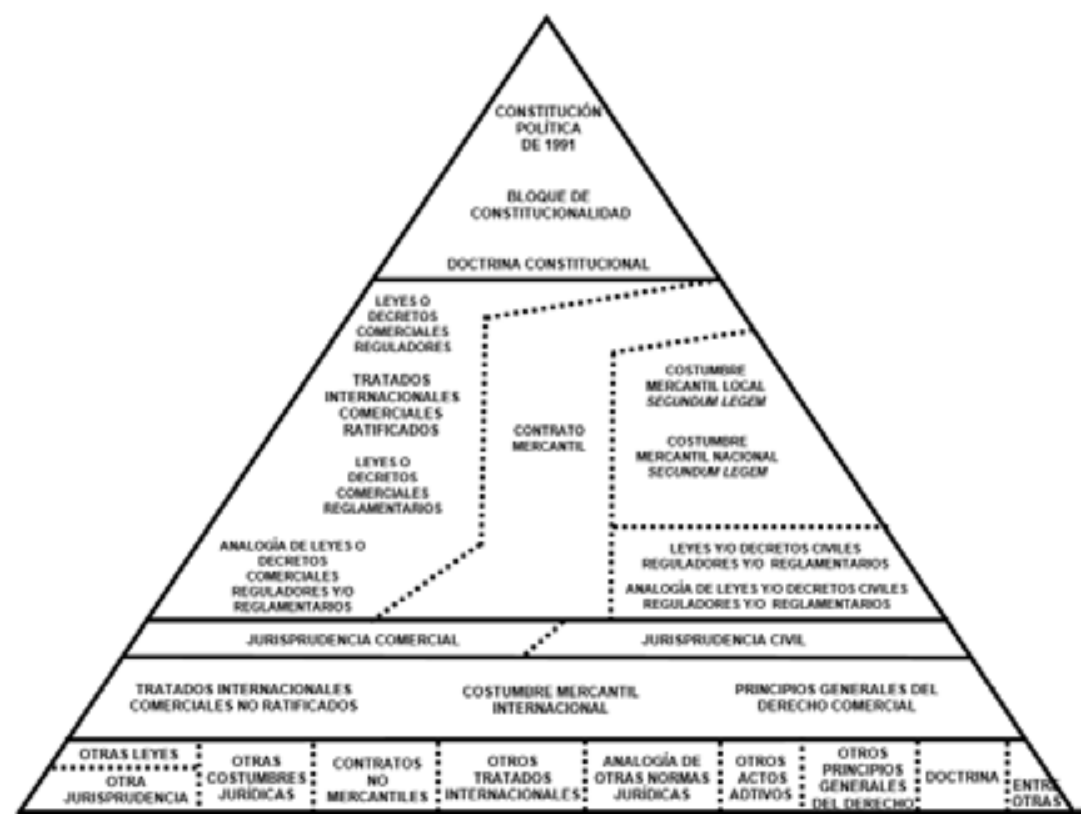

Fuente: Elaboración propia con base en Perilla, 2015a. 
Se evidencia de esta forma que los tratados internacionales sobre derecho comercial que son ratificados por Colombia, hacen parte íntegra de la ley y se ubican entre las leyes comerciales reguladoras y reglamentarias. La ubicación media implica que en algunas ocasiones el tratado ratificado puede tener la función de establecer un marco normativo (regulación) que exige una posterior reglamentación por las normas internas (Ureña, 2012). También puede darse el caso en el cual los tratados comerciales de derecho internacional ratificados sean la reglamentación de una norma reguladora interna (Ureña, 2015), por lo cual se ubica en un punto medio que pueda facilitar cualquiera de las dos funciones.

Este ajuste a la pirámide de fuentes del derecho comercial implica que los cambios normativos generados o exigidos por tratados internacionales de derecho comercial tienen la capacidad para derogar parcial o totalmente las normas internas de igual nivel (Guardiola, 1998; Pérez, 2004); los tratados internacionales reguladores pueden derogar normas reguladoras o reglamentarias, mientras que aquellos reglamentarios pueden derogar o exigir cambios en las leyes internas que cumplen con esta función. En el caso del tratado de libre comercio celebrado entre Colombia y Estados Unidos, se trata de una norma con carácter regulador y en su contenido se hacen una serie de exigencias tendientes a modificar parcialmente el contrato de agencia mercantil, implicando que desde el monismo impuro se presenta una posible derogatoria del Código de Comercio en el sentido que será expuesto a continuación.

\section{Cambios en los elementos contractua- les de la agencia mercantil a partir del Tratado de Libre Comercio celebrado entre Colombia y Estados Unidos}

El tratado de libre comercio celebrado entre Colombia y Estados Unidos fue suscrito por ambos Estados y aprobado en Colombia a través de leyes del Congreso de la República, implicando que desde el monismo impuro las normas internacionales hacen parte íntegra del sistema jurídico colombiano en calidad de ley comercial. En el anexo 11-E de esta norma internacional, ambos países convinieron los siguientes compromisos relacionados con la agencia mercantil ${ }^{18}$ :

- Modificar o derogar las cesantías comerciales de las que habla el primer inciso del artículo 1324 del Código de Comercio, es decir, el pago de la doceava parte calculada en los últimos tres años o el tiempo menor que corresponda.

- Modificar o derogar la indemnización a la finalización del contrato de la cual habla el segundo inciso del artículo 1324 del Código de Comercio, es decir, aquella que se configura por causa injustificada por parte del agenciado y debe ser pagada al agente. Por el contrario, se exige la aplicación de principios generales del derecho, como lucro cesante y daño emergente para el caso colombiano.

- Modificar o derogar la exclusividad a favor del agente que se exige del agenciado, para que se constituya en un elemento accidental del contrato.

Todas estas modificaciones o derogatorias debían ser aplicadas por ambos Estados en un plazo de seis meses, contados a partir de la entrada en vigor del Tratado de Libre Comercio entre Colombia y Estados Unidos, es decir, el 15 de mayo de 2012; el plazo para esta adaptación venció en noviembre 15 de 2012 y es relevante señalar que el Congreso de la República de Colombia no adelantó ninguna actividad legislativa relacionada. Por esta razón, en el Código de Comercio siguen estando expresamente vigentes la cesantía, la indemnización por terminación y la exclusividad a favor del agente por parte del agenciado.

Sin embargo, si se tiene en cuenta que el Tratado de Libre Comercio entre Colombia y 
Estados Unidos fue aprobado a través de una ley expedida por el Congreso de la República, se trata de una norma jurídica, del mismo nivel que el Código de Comercio, que incluye una modificación legal. Considerando que esta norma posterior daba un plazo perentorio para la entrada en vigencia de los cambios exigidos y acordados por ambas partes frente a la agencia comercial, se debe afirmar que se configuró una derogatoria aprobada por el Congreso respecto de estas disposiciones normativas aplicables al contrato. Sobre la derogatoria de normas se pronuncia la Corte Constitucional asi ${ }^{19}$ :

La derogación tiene como función dejar sin efecto el deber ser de otra norma, expulsándola del ordenamiento. Por ello se ha entendido que la derogación es la cesación de la vigencia de una disposición como efecto de una norma posterior, que no se fundamenta en un cuestionamiento sobre la validez de la normas, por ejemplo, cuando es declarada inexequible, sino en criterios de oportunidad libremente evaluados por las autoridades competentes, $y$ en especial, en relación con las leyes por el Congreso. Así la derogación no deriva de conflictos entre normas de distinta jerarquía sino de la libertad política del legislador. La derogación no afecta tampoco ipso iure la eficacia de la norma derogada, pues en general las situaciones surgidas bajo su vigencia continúan rigiéndose por ella, por lo cual la norma derogada puede mantener su eficacia, la cual poco a poco se va extinguiendo (Sentencia C-901 de 2011).

Se hace evidente que según la teoría del monismo impuro, las normas internacionales aprobadas por el Congreso de la República ocupan el mismo nivel de las leyes comerciales como el Código de Comercio y en este sentido se puede hablar de derogatorias. Este ejercicio de derogatoria tiene dos alcances, el primero referente a la derogatoria expresa, a saber:

La derogación expresa el legislador determina de manera precisa el o los artículos que retira del ordenamiento, por lo que no se hace necesaria ninguna interpretación, ya que simplemente se cumple una función de exclusión desde el momento que así se establezca. La derogación orgánica refiere a cuando la nueva ley regula integralmente la materia, que en términos de la Corte Suprema de Justicia supone "que la nueva ley realiza una mejora en relación con la ley antigua; que aquella es más adecuada a la vida social de la época y que, por tanto, responde mejor al ideal de justicia, que torna urgente la aplicación de la nueva ley;(...) que por lo mismo debe ser lo más amplia posible para que desaparezcan las situaciones que el propio legislador ha querido condenar y evidentemente arrasó con la ley nueva” (Sentencia C-901 de 2011).

El segundo alcance es la derogación tácita, delimitada por la Doctrina Constitucional así:

La derogación tácita obedece a un cambio de legislación, a la existencia de una incompatibilidad entre la ley anterior y la nueva ley, lo cual hace indispensable la interpretación de ambas leyes para establecer la vigente en la materia o si la derogación es parcial o total. Tiene como efecto limitar en el tiempo la vigencia de una norma, es decir, suspender su aplicación y capacidad regulatoria, aunque en todo caso el precepto sigue amparado por una presunción de validez respecto de las situaciones ocurridas durante su vigencia. Cuando se deroga tácitamente una disposición, no se está frente a una omisión del legislador sino que al crear una nueva norma ha decidido que la anterior deje de aplicarse siempre que no pueda conciliarse con la recientemente aprobada (Sentencia C-901 de 2011).

Aplicando estos preceptos jurídicos al caso de las disposiciones del Tratado de Libre Comercio celebrado entre Colombia y Estados Unidos referentes a la agencia comercial, se hace evidente que en un primer momento los Estados contemplaron la posibilidad de adelantar una derogatoria expresa. Sin embargo, por la ausencia de trabajo legislativo en este sentido y para dar cumplimiento al plazo de seis meses, se configuró una derogatoria implícita dada por normas del mismo nivel.

Para que sea posible la derogación debe darse por otra de igual o superior jerarquía. Entonces, 
la derogación tácita es aquella que surge de la incompatibilidad entre la nueva ley y las disposiciones de la antigua, que suele originarse en una declaración genérica en la cual se dispone la supresión de todas las normas que resulten contrarias a la expedida con ulterioridad (Sentencia C-901 de 2011).

Considerando que las disposiciones referentes a la agencia comercial contenidas en el
Tratado de Libre Comercio son incompatibles con el Código de Comercio, y son normas del mismo nivel, los elementos naturales identificados a propósito de la agencia comercial fueron modificados, pasando a ser elementos accidentales, y otros fueron suprimidos definitivamente del Código de Comercio. En atención a este razonamiento, en la Tabla 2 se presenta un paralelo de los elementos contractuales antes y después del Tratado de Libre Comercio:

Tabla 2. Elementos contractuales de la agencia mercantil antes y después del TLC Colombia - Estados Unidos.

\begin{tabular}{|c|c|c|}
\hline & Antes del TLC Colombia - EEUU & Después del TLC Colombia - EEUU \\
\hline $\begin{array}{l}\text { ELEMENTOS } \\
\text { ESENCIALES }\end{array}$ & $\begin{array}{l}\text { Encargo de promover y explotar negocios } \\
\text { Independencia del agente } \\
\text { Estabilidad } \\
\text { Actuación del agente por cuenta del agenciado } \\
\text { Área de negocio específica } \\
\text { Zona geográfica delimitada }\end{array}$ & $\begin{array}{l}\text { Encargo de promover y explotar negocios } \\
\text { Independencia del agente } \\
\text { Estabilidad } \\
\text { Actuación del agente por cuenta del agenciado } \\
\text { Área de negocio específica } \\
\text { Zona geográfica delimitada }\end{array}$ \\
\hline $\begin{array}{l}\text { ELEMENTOS } \\
\text { NATURALES }\end{array}$ & $\begin{array}{l}\text { Remuneración a favor del agente } \\
\text { Exclusividad a favor del agente } \\
\text { Prestaciones a la terminación del contrato }\end{array}$ & Remuneración a favor del agente \\
\hline \multirow{2}{*}{$\begin{array}{l}\text { ELEMENTOS } \\
\text { ACCIDENTALES }\end{array}$} & Término fijo o indefinido & Término fijo o indefinido \\
\hline & Exclusividad a favor del agenciado & Exclusividad a favor del agenciado y del agente. \\
\hline
\end{tabular}

Fuente: elaboración propia.

Se evidencia de esta forma que como consecuencia de la entrada en vigor del Tratado de Libre Comercio entre Colombia y Estados Unidos, la agencia comercial sigue existiendo en el sistema jurídico colombiano. Esto se debe a que los elementos de la esencia, que se constituyen en requisitos de existencia, continúan siendo los mismos. Sin embargo, existe una modificación considerable en los elementos naturales del contrato, a saber: aunque el contrato continúa siendo por naturaleza oneroso, la exclusividad a favor del agente pasa a ser un elemento accidental; en caso de no señalar expresamente nada, se presume que no se presenta exclusividad ni a favor del agente ni del agenciado.
Por su parte, el compromiso de inaplicación de la cesantía comercial de la cual trata el primer inciso del artículo 1324 del Código de Comercio y la indemnización contenida en el segundo inciso de la misma norma, generan la derogatoria implícita total de estas normas. Tan es así, que en el mismo tratado internacional se acuerda aplicar en su reemplazo las instituciones jurídicas generales de lucro cesante y daño emergente para el caso colombiano. Se manifiesta de esta forma un primer caso de derogatoria implícita entre normas internacionales y nacionales, que en caso de conflicto entre comerciantes de ambos países, se deberá solucionar en las jurisdicciones contractuales desde la teoría del monismo impuro. 
Por último, corresponde preguntarse si este proceso de derogatoria implícita solamente es aplicable a los contratos de agencia mercantil celebrados en el marco del tratado entre Colombia y Estados Unidos. Sobre el particular debe considerarse que en virtud de los principios constitucionales del sistema jurídico actual, específicamente en los referentes a la igualdad materializada en la consolidación de normas generales y abstractas, estos cambios normativos deberían aplicarse a todos los sujetos que se rijan por las normas comerciales colombianas referentes a la agencia mercantil. Sin embargo, se trata de un tema que hasta ahora empieza a hacerse evidente y en el momento en que inicien los litigios entre comerciantes, serán los jueces quienes desde sus razonamientos contribuyan a apoyar o debatir la hipótesis que desde la teoría jurídica acaba de ser probada en este trabajo académico.

\section{Conclusiones}

El contrato de agencia mercantil en Colombia ha suscitado diversos debates académicos desde su inclusión en el ordenamiento jurídico colombiano. Tales debates han permitido delimitar los elementos contractuales de este negocio jurídico y agruparlos según sean esenciales, naturales o accidentales. Los elementos esenciales de la agencia mercantil son: el encargo de promover o explotar un negocio de forma independiente, con estabilidad en el tiempo, actuando por cuenta de otro y con un giro de los negocios delimitado en una zona del territorio nacional prefijada. Son esenciales dado que a falta de los mismos, deviene el contrato en inexistente o se configura uno totalmente diferente.

Por su parte, se han identificado tres elementos de la naturaleza al respecto de los cuales se puede pactar en contrario: remuneración a favor del agente; exclusividad a favor del agente y en cabeza del agenciado; y unas indemnizaciones al finalizar el contrato. Estas indemnizaciones son: primero, la cesantía comercial a favor del agente de un porcentaje específico de lo recibido por el agenciado; y segundo, la indemnización por causas injustificadas por parte del agenciado. Estos elementos se presumen contratados a falta de pacto expreso en contrario por parte de los comerciantes vinculados contractualmente.

Por último, los dos elementos accidentales de la agencia comercial son los que se presentan si las partes así lo disponen expresamente al momento de celebrar el negocio jurídico: el término fijo o indefinido del contrato, y la exclusividad a favor del agenciado y en cabeza del agente. Quiere decir entonces que si las partes no señalan expresamente estos dos elementos, se entienden como no incluidos en la agencia mercantil.

Ahora bien, algunos de los elementos antes identificados fueron objeto de debate durante las negociaciones que llevaron a la aprobación definitiva del tratado de libre comercio entre Colombia y Estados Unidos. Como consecuencia de estas negociaciones, ambos Estados acordaron modificar o derogar, en un plazo máximo de seis meses luego de la entrada en vigor del tratado, los siguientes elementos: i) la cesantía comercial incluida en el primer inciso del artículo 1324 del Código de Comercio; ii) la indemnización incluida en el segundo inciso del artículo 1324 del Código de Comercio; y iii) la exclusividad a favor del agente y en cabeza del agenciado en los casos de agencia mercantil.

Pese a este compromiso, Colombia no cumplió con tal obligación y el Congreso de la República no adelantó ningún tipo de trabajo legislativo sobre el tema. Una vez vencido el plazo, surge la cuestión en torno a si las normas objeto de debate siguen o no vigentes en el sistema jurídico colombiano actual. Para solucionar esta controversia, se consulta la pirámide de fuentes del derecho comercial, aplicable actualmente desde la perspectiva del antiformalismo. $\mathrm{El}$ antiformalismo es la iusteoría imperante actualmente en Colombia, pues reconoce que el 
derecho tiene una naturaleza abierta y debe ser complementado por múltiples fuentes a través de interpretaciones auténticas.

El antiformalismo como iusteoría avala entonces la posibilidad de que normas como el derecho internacional hagan parte íntegra de las normas de derecho doméstico. Así, se formula la teoría del monismo impuro como una conversión de las teorías europeas desde el contexto particular de Colombia. Esta nueva clase de monismo comprende que los tratados internacionales comerciales hacen parte íntegra de las leyes comerciales, pues el legislador aprueba los mismos a través de una norma de rango legal. Esta inclusión de las normas internacionales en las nacionales, permite que las primeras tengan una incidencia directa sobre el contenido de las segundas y puedan generar modificaciones específicas en casos puntuales.

En el caso del contrato de agencia comercial, su regulación inicial se encuentra en el Código de Comercio que es una norma de rango legal con funciones reguladoras y reglamentarias. Los compromisos formulados en el tratado de libre comercio celebrado entre Colombia y Estados Unidos fueron aprobados por el Congreso de la República a través de normas legales del mismo nivel que las comerciales. Por esta razón, las normas posteriores sobre agencia mercantil están llamadas a modificar o derogar tácitamente las normas originarias sobre agencia mercantil.

En este sentido, y materializando los principios antiformalistas de la interpretación auténtica, el contrato de agencia comercial se encuentra actualmente modificado: aunque se conservan los elementos de la esencia, los elementos naturales fueron reformados sustancialmente. De manera particular, deja de ser elemento esencial la exclusividad a favor del agente y se entiende ahora como elemento accidental que exige ser pactado expresamente para ser incluido en el negocio jurídico. Además, las prestaciones económicas contempladas en el ar- tículo 1324 del Código de Comercio son derogadas tácitamente, pues no habrá lugar a exigir cesantía comercial ni indemnización especial por terminación sin justa causa. Por el contrario, se requiere aplicar las instituciones tradicionales del lucro cesante y del daño emergente en casos de terminación unilateral.

Retomando todo lo anterior, el presente trabajo académico ha planteado una propuesta desde las exigencias antiformalistas del sistema jurídico colombiano actual, la cual está llamada a ser probada o debatida cuando se empiecen a configurar pleitos entre comerciantes nacionales y extranjeros en el marco del tratado de libre comercio entre Colombia y Estados Unidos. Será responsabilidad de los operadores jurídicos, a través de su labor, generar interpretaciones para garantizar los derechos de las partes gracias a una elucidación armónica de las fuentes del derecho mercantil.

\section{Notas}

${ }^{1}$ Sobre el particular se refiere la Doctrina así: "suele atribuirse al derecho mercantil la característica de la especialidad y al mismo tiempo la de la universalidad. La primera característica se le atribuye en el ámbito estatal con respecto a la restante normativa del Estado, y en el más específico ámbito del derecho privado con respecto al derecho civil. La siguiente característica le viene conferida a nivel supraestatal, a causa de su reconocida fuerza expansiva, como derecho uniforme más allá de las fronteras nacionales" (Galgano, 1980, p. 23).

${ }^{2}$ El artículo 1501 del Código Civil colombiano se refiere a los requisitos esenciales, naturales y accidentales del negocio jurídico, así: "se distinguen en cada contrato las cosas que son de su esencia, las que son de su naturaleza, y las puramente accidentales. Son de la esencia de un contrato aquellas cosas sin las cuales, o no produce efecto alguno, o degeneran en otro contrato diferente; son de la naturaleza de un contrato las que no siendo esenciales en 
él, se entienden pertenecerle, sin necesidad de una cláusula especial; y son accidentales a un contrato aquellas que ni esencial ni naturalmente le pertenecen, y que se le agregan por medio de cláusulas especiales".

${ }^{3}$ La jurisprudencia ha señalado que no es posible limitarse a un acto o contrato específico, pues se requiere una serie coordinada de los mismos. A manera de ejemplo, la Corte Suprema de Justicia se refiere a la reventa, así: "este aspecto aleja a la agencia comercial sustancialmente de los vínculos en que el intermediario adquiere los productos para la reventa, en los cuales éste, en uso de sus habilidades, saca provecho de la diferencia de precios de compra y enajenación, corriendo los riesgos de cartera propios de quien ejerce actividades de comercio" (Sentencia del 10 de septiembre de 2013).

${ }^{4}$ Sobre el particular se señala: "esa independencia se traduce, en la práctica, en la apertura de oficinas o establecimientos de comercio por parte del agente, la vinculación de empleados calificados, la selección de las técnicas de mercadeo y de publicidad y, en general, en la realización de actos encaminados a procurar el cumplimiento del encargo en las circunstancias de modo, tiempo y lugar que hayan sido convenidas" (Giraldo, 2012, p. 7).

5 Al respecto, Giraldo (2012) señala que "El agente decide, en ejercicio de su independencia, el modo, el tiempo o la cantidad de su actividad, lo cual significa que no se encuentra sujeto a horarios de trabajo o a itinerarios. Pero, por ejemplo, no riñe con ella que el empresario le exija al agente un número determinado de personal para realizar su labor, o con que el agenciado pueda establecer algunas de las condiciones de la distribución de los bienes que se encargan al agente; así, ordinariamente es él quien fija las condiciones de venta en cuanto a los precios a que el agente puede distribuir el producto, o las condiciones de pago que se pueden conceder a quienes adquieren el producto" (p. 8).
${ }^{6}$ Se debe advertir que la agencia mercantil no exige un lapso de tiempo específico, pues dependerá de cada caso en concreto. A manera de ejemplo: existen agencias que se presentan según la temporada o festividades específicas, por lo cual la estabilidad se evalúa en función de ese lapso de tiempo particular. Sobre el tema, se refiere la jurisprudencia así: "tiene un ánimo de estabilidad o permanencia, en la medida que se refiere a la promoción continua del negocio del agenciado y no a un asunto en particular, lo que excluye de entrada los encargos esporádicos y ocasionales" (Sentencia del 10 de septiembre de 2013).

${ }^{7}$ Se debe advertir que este elemento ha sido debatido en la doctrina para determinar si es o no esencial del contrato de agencia. El debate principal radica en que no se incluye el mismo en la redacción del artículo y su posterior desarrollo se ha dado en la jurisprudencia. Sin embargo, por el precedente establecido por la Corte Suprema de Justicia, actualmente se presenta cierto nivel de aceptación doctrinario sobre su calidad de esencial. Sobre el particular, se pueden consultar las siguientes obras: Arrubla (1997), Bonivento (1999), Cárdenas (1984), Giraldo (2012), Gómez (1980), Namén (1995), Valencia (1985) y Vallejo (1999).

${ }^{8}$ Sobre los riesgos para ambas partes se ha pronunciado la doctrina de este modo: "Consideramos que los siguientes riesgos están a cargo del empresario en un contrato de agencia mercantil: los de colocación o no de los bienes o mercancías, los de cartera, los de su pérdida fortuita. Por su parte, los principales riesgos a cargo del agente-distribuidor serían los del sostenimiento de la infraestructura necesaria para la distribución, que muchas veces se referirá a los establecimientos de comercio necesarios para desarrollar dicha labor" (Giraldo, 2012, p. 14).

${ }^{9}$ Sobre el asunto, así se refiere la jurisprudencia: "No obstante la autonomía de que goza la agencia, la característica mercantil interme- 
diadora, lo hace afín con otros contratos, con los cuales puede concurrir, pero sin confundirse con ninguno de ellos, ya que tiene calidades específicas que, por lo mismo, lo hacen diferente, razón por la cual, su demostración tendrá que ser inequívoca. De suerte, que una persona bien puede recibir estos encargos mediante dichos contratos y no ser agente comercial, pero dentro de aquella actividad también puede la misma recibir el especial de promover y explotar los negocios del empresario ora como representante o agente, pero en virtud de un contrato de agencia" (Sentencia del 4 de abril de 2008).

${ }^{10}$ Se habla de posibles prestaciones porque no siempre están llamadas a configurarse, denotando así que no se trata de un elemento de la esencia sino de un elemento que se presume y que admite pacto o situación en contrario.

${ }^{11}$ Parte de la doctrina cuestionaría esta inclusión afirmando que se trata de un derecho y una obligación, pero que no se trata de un elemento de la naturaleza. Sin embargo, otros elementos esenciales y naturales también son derechos y obligaciones, como el caso de la exclusividad a favor del agente, la estabilidad, la independencia, entre otros. Así, se trata de una interpretación auténtica.

${ }^{12}$ La iusteoría antiformalista fue establecida por la Constitución Política de 1991 y responde al fracaso del Estado colombiano liderado por la Constitución Nacional de 1886 (Moncada, 2007). Bajo la Constitución Nacional de 1886, el sistema jurídico estaba marcado por una iusteoría formalista; "la historia no es nueva: formalismo y antiformalismo son las posiciones extremas y siempre recurrentes entre las que oscila el péndulo del derecho" (Bobbio, 1995, p. 12). La pretensión principal del formalismo era comprender al derecho como un entramado de normas perfectas, que desde una perspectiva positivista intenta proponer la inexistencia de lagunas (Lemaitre, 2009). Esta perfección se justifica desde argumentos iusnaturalistas, según los cuales las leyes están hechas por el legislador tradicional a través de ejercicios inspirados en realidades preexistentes al hombre (Cely, 2012). Esta perfección fundamentada en la labor legislativa, lleva a que el operador jurídico esté llamado a realizar interpretaciones miméticas (Perilla, 2013). Estas interpretaciones miméticas vinculan métodos formalistas como la exégesis (Vernengo, 1977), el historicismo (Savigny, 1979) y el teleológico (Castillo \& Luján, 2006). Sobre estos métodos de interpretación se recomienda consultar la obra de Herrera (1994) y López Medina (2006a; 2006b).

13 Sobre el término régimen jurídico, Perilla (2015b) señala: “en el presente trabajo se entiende por ordenamiento jurídico el conjunto de sistemas jurídicos. Cada sistema jurídico es un conjunto de normas organizadas de manera jerárquica, encabezadas por una Constitución particular. Cuando estas normas se agrupan por áreas (civil, laboral, propiedad intelectual, etc.) constituirán regímenes jurídicos" (p. 5). A su vez, Bercholc (2014) hace énfasis en que los regímenes jurídicos tendrán enfoques que en su mayoría son determinados por las normas superiores. Para profundizar en el tema se recomienda consultar la obra de Bobbio (2007).

${ }^{14} \mathrm{La}$ estructura propuesta como nueva pirámide de fuentes del derecho comercial encuentra su justificación así: "La Constitución es entendida como norma de normas, la cual no se limita a ser un documento estático, sino que se complementa de manera dinámica y permanente por el Bloque de Constitucionalidad y las interpretaciones que hace la Corte Constitucional en calidad de Doctrina. A nivel legal, el régimen jurídico comercial reconoce cuatro fuentes en el mismo nivel, pero con jerarquías internas diferentes. Revisten igual importancia la ley comercial, el contrato mercantil, la costumbre comercial y la ley civil, significando que cualquier caso puede ser resuelto por cualquiera de ellas a manera de fuente principal. Ahora bien, el orden para estudiar estas fuentes es: primero, las leyes y decretos comerciales que regulan materias es- 
pecíficas, para luego consultar aquellos que las reglamentan o las solucionan por analogía. En caso en que la Ley comercial sea insuficiente para solucionar un caso particular, se podrá acudir al contrato mercantil, el cual es ley para las partes y puede ser aplicado desde el contenido de cada una de sus cláusulas. Si las normas anteriores no fuesen suficientes, se puede aplicar la costumbre mercantil (primero la local y luego la nacional) que esté contenida en Resoluciones Administrativas de la Cámara de Comercio. Finalmente, se podrá acudir a manera de norma supletiva a la ley civil y a su analogía. En caso de duda o vacío en cualquiera de las fuentes anteriores, es posible acudir primero a la jurisprudencia comercial y luego a la civil. En caso de necesidad se puede acudir a tratados internacionales comerciales que hayan sido ratificados por Colombia. Acto seguido, cuentan con igual jerarquía los tratados internacionales mercantiles no ratificados por Colombia, la costumbre mercantil internacional y los principios generales del derecho comercial. Por último, y sólo en caso en que las anteriores normas sean insuficientes para resolver situaciones con relevancia mercantil, es posible acudir a las demás fuentes del derecho, que para estos efectos son entendidas del mismo nivel" (Perilla, 2015a, p. 21).

${ }^{15}$ Sobre el monismo impuro se señala que "reconoce que tanto el derecho nacional como el internacional deben interactuar de forma dinámica independiente de su fuente jurídica, ubicando las normas en el mismo nivel y generando complementos jurídicos inmediatos. Así, las normas nacionales e internacionales que traten sobre los mismos temas jurídicos deberán ser una sola, pues las teorías antiformalistas del derecho reconocen que los sistemas jurídicos son abiertos y han de ser complementados por múltiples fuentes a través de interpretaciones auténticas permanentes. El mismo impuro trasciende las jerarquías establecidas por las dos vertientes del monismo, pues no es dable acudir primero a una y otra norma, sino que ellas se deben interpretar sistemática y simultáneamente. Este rompimiento de los parámetros establecidos para el monismo desde las teorías tradicionales analizadas, se da como consecuencia de la consolidación de una Teoría Transnacional del Derecho" (Perilla, 2016, pp. 405-406).

${ }^{16}$ López (2004) se refiere a los trasplantes como aquel proceso hermenéutico que se hace en contextos diferentes a aquellos en los cuales fue creada una teoría y que generan mutaciones desde el nuevo contexto en el cual se aborde el tema. Así, por ejemplo, las teorías del derecho son gestadas en sitios de producción como Europa y Estados Unidos, pero cuando las mismas son leídas desde sitios de recepción como Colombia, carecen de los elementos contextuales suficientes para entender la teoría en su totalidad. Esto implica que los sitios de recepción harán la lectura de las teorías desde su propio contexto, añadiendo nuevos elementos que pueden ser tachados como incorrectos por parte de los sitios de producción. Estas lecturas complementadas desde los sitios de recepción configurarán un proceso de transmutación de teorías, según el cual la teoría creada originalmente sufre variaciones que la hacen diferente a la original.

${ }^{17}$ El término misreading es utilizado por Bloom (1995), quien sugiere que las lecturas descontextualizadas generan cambios en las teorías que permiten la consolidación de una nueva. Así, aunque las lecturas generadas desde los sitios de recepción podrían ser tachadas como incorrectas por los productores, lo que sucede en realidad es que se trata de una construcción útil para el nuevo contexto en el cual son aplicadas. Así, en caso de tener aceptación social general, los sitios de recepción pueden generar nuevas teorías (López-Medina, 2004; Perilla, 2015a).

${ }^{18}$ El compromiso específico incluido en el anexo 11-E del aprobado Acuerdo de Promoción Comercial entre la República de Colombia y Estados Unidos de América, señala: "1. Si una Parte mantiene una medida en el nivel central de gobierno: a) otorgando un derecho al agente a que a la terminación de un contrato de agencia 
comercial el principal le pague una suma equivalente a una porción de la comisión, regalía o utilidad recibida por el agente en virtud del contrato; b) requiriendo que en el evento en que el principal termine un contrato de agencia comercial sin justa causa o el agente termine un contrato de agencia comercial por justa causa probada por el principal, el principal deba pagar una indemnización equitativa al agente como retribución a los esfuerzos del agente para acreditar la marca, la línea de productos o los servicios objeto de un contrato de agencia comercial; o c) estableciendo que un contrato de agencia comercial crea una agencia exclusiva salvo pacto en contrario; esa parte deberá modificar la medida o derogarla de conformidad con el párrafo 2, dentro de los seis meses siguientes a la entrada en vigencia de este Tratado. 2. Una Parte deberá: a) modificar una medida descrita en el párrafo 1(a) haciendo que el otorgamiento del derecho al pago, sea inaplicable a las partes de un contrato de agencia comercial; b) modificar una medida descrita en el párrafo 1(b) haciendo el requerimiento de pagar la indemnización equitativa inaplicable a las partes de un contrato de agencia comercial y en su lugar, hacer que cualquier indemnización derivada de la terminación por parte del principal de un contrato de agencia comercial sin justa causa, o derivada de la terminación de un contrato de agencia comercial por parte del agente por justa causa provocada por el principal, sea determinada de conformidad con (i) los principios generales del derecho contractual (por ejemplo, daño emergente, lucro cesante y detrimental reliance); $\mathrm{y}$, en el evento en que las partes lo estipulen así expresamente, ii) las estipulaciones voluntariamente acordadas entre el principal y el agente en un contrato de agencia comercial, en la medida que sean compatibles con las leyes aplicables. c) modificar una medida descrita en el párrafo 1(c) estableciendo que un principal pueda contratar más de un agente en una misma zona geográfica, para el mismo ramo de actividades o productos, salvo que el contrato de agencia comercial disponga lo contrario. 3. Nada en este Anexo impedirá que continúe la aplicación, en la medida en que sea requerida la Constitución de una de las Partes, de una medida descrita en el párrafo 1 (a) o (c) a contratos de agencia comercial celebrados antes de la entrada en vigencia de la legislación que se adopte para implementar este Anexo. 4. Una Parte no adoptará una medida descrita en el párrafo 1.5. Para efectos de este Anexo, contrato de agencia comercial significa, (a) para Colombia, un contrato de agencia comercial según lo consagrado en los artículos 1317 a 1331 del Código de Comercio de Colombia; solo cuando el contrato esté relacionado con bienes comerciales, y (b) para Estados Unidos, cualquier contrato en que una parte acuerde distribuir bienes comerciales para otra parte".

${ }^{19}$ Para definir estos conceptos, el presente estudio se vale de la jurisprudencia de la Corte Constitucional, pues se trata de la guardiana e intérprete de la Constitución por excelencia y sus pronunciamientos gozan de especial jerarquía en el sistema jurídico colombiano actual (LópezMedina, 2006a). Perilla (2015c) coincide con esta valoración: "la Corte Constitucional tiene la tarea de asegurar que la Constitución sea un cuerpo normativo vivo que responda de forma permanente a las necesidades que se gestan en el conglomerado social (Arango, 1993). Se establece así que el legislador tradicional ya no es la autoridad más importante en el sistema jurídico, sino que es la Corte Constitucional quien interpreta las normas existentes e incluso puede crear otras para garantizar la vida de la Constitución" (p. 8). Sobre la prevalencia de la Corte Constitucional también pueden ser consultadas las obras de Bonilla (2006; 2009), García (2008), Olano (2010) y Rodríguez (1999).

\section{Referencias}

Angarita, J. (2005). Lecciones de Derecho Civil. Bogotá: Temis.

Arango, R. (1993). Jurisdicción e interpretación constitucional. Revista de Derecho Público, 4, 31-38. 
Arrubla, J. A. (1997). Contratos Mercantiles (Tomo I). Bogotá: Editorial Jurídica Diké.

Azuero, A. (2009). Redes de diálogo judicial trasnacional: una aproximación empírica al caso de la Corte Constitucional. Revista de Derecho Público Universidad de los Andes, 22. Recuperado de https://derechopublico. uniandes.edu.co/components/com_revista/ archivos/derechopub/pub98.pdf

Benvenisti, E. (2008). Reclaiming Democracy: The Strategic Uses of Foreign and International Law By National Courts. American Journal of International Law, 102, 241-274.

Bercholc, J. (2014). Temas de Teoría del Estado. Buenos Aires: La Ley.

Bloom, H. (1995). A map of Misreading. Oxford University Press.

Bobbio, N. (1995). El problema del positivismo jurídico. México: Distribuciones Fontamara.

Bobbio, N. (2007). Teoría general del derecho. Bogotá: Editorial Temis.

Bogdandy, A. (2008). Pluralism, direct effect, and the Ultimate Say: On the Relationship between International and Domestic Constitutional Law. International Journal of Constitutional Law, 6(3-4), 397-413. doi: 10.1093/icon/mon015

Bonilla, D. (2006). La Constitución multicultural. Bogotá: Siglo del Hombre Editores.

Bonilla, D. (2009). Teoría del Derecho y Transplantes jurídicos. Bogotá: Siglo del Hombre Editores.

Bonivento, J. A. (1999). Contratos mercantiles de intermediación: representación, mandato, comisión, preposición, agencia comercial, corretaje. Bogotá: Ediciones Librería del Profesional.

Brownlie, I. (2008). Principles of Public International Law. Oxford: Oxford University Press.

Butler, W. (1985). Comparative Approaches to International Law. Academy of International Law at the Hague, Recueil des Cours, 190, 9-90.

Cárdenas, J. P. (1984). El contrato de Agencia Mercantil. Bogotá: Editorial Temis; Colegio Mayor Nuestra Señora del Rosario.

Castillo,J.L., \& Luján, M.(2006). Razonamiento Judicial: Interpretación, argumentación y motivación de las resoluciones judiciales. Perú: Ara Editores.

Castro de Cifuentes, M. (2009). Derecho comercial: actos de comercio, empresas, comerciantes y empresarios. Bogotá: Ediciones Uniandes y Temis.

Cely, L. A. (2012). El carácter especulativo de la iusteoría periférica. Revista Prolegómenos. Derechos y Valores, 17(34), 3341.

Constitución Política (1886). Consejo Nacional Constituyente. Colombia.

Constitución Política (1991). Congreso de la República de Colombia. Colombia.

Cuberos, F. (2005). Agencia comercial: entre la comparación y la exégesis. Revista de Derecho Privado, 35, 1-33. Recuperado de http://www.redalyc.org/ html/3600/360033183001/

Decreto 410 de 1971. Por el cual se expide el Código de Comercio. Diario Oficial No. 
33.339. Presidencia de la República, marzo de 1971.

Escobar, G. (s.f.). Negocios civiles y comerciales I: negocios de sustitución. Bogotá: Editorial Universidad Externado de Colombia.

Galgano, F. (1980). Historia del derecho mercantil. Barcelona: Laia.

García, L. (2008). El "nuevo" derecho en Colombia: ¿Entelequia innecesaria o novedad pertinente? Revista de Derecho, 29, 289-330.

Giraldo, C. J. (2012). La agencia comercial en el derecho colombiano. Revista de Derecho Privado, 47, 1-31. Recuperado de http://www.redalyc.org/ html/3600/360033218006/

Gómez, J. A. (1980). Contratos mercantiles. Bogotá: Editorial Anaké.

Guardiola, E. (1998). Contratos de colaboración en el comercio internacional. Barcelona.

Herrera, F. (1994). Filosofía del derecho. Bogotá: Universidad Javeriana.

Hinestrosa, F. (2015). Tratado de las obligaciones II: de las fuentes de las obligaciones el negocio jurídico. Bogotá: Universidad Externado de Colombia.

Kelsen, H. (1952). Principles of International Law. Nueva York: Rinehart \& Co.

Kelsen, H. (2009). Teoría Pura del Derecho. Buenos Aires: Eudeba.

Lemaitre, J. (2009). El derecho como conjuro: fetichismo legal, violencia y movimientos sociales. Bogotá: Siglo del Hombre Editores; Universidad de los Andes.
Ley 57 de 1887. Con arreglo al artículo 52 de la Constitución de la República, declárase incorporado en el Código Civil el Título III (arts. 19-52) de la misma Constitución. Diario Oficial No. 7.019. Congreso de la República de Colombia, abril de 1887.

Ley 1143 de 2007. Por medio de la cual se aprueba el "Acuerdo de promoción comercial entre la República de Colombia y los Estados Unidos de América", sus "Cartas Adjuntas" y sus "Entendimientos", suscritos en Washington el 22 de noviembre de 2006. Diario Oficial No. 46.679. Congreso de la República, julio de 2007.

López-Álvarez, A.L. (2011). El contrato de agencia comercial y los cambios introducidos en la negociación del TLC entre Estados Unidos y los países andinos. Revista e-mercatoria, 10(1). Recuperado de http://revistas.uexternado. edu.co/index.php/emerca/article/ view/2905/2546

López-Guzmán, F. (2007). Introducción al derecho mercantil. Bogotá: Temis.

López-Medina, D. E. (2004). Teoría impura del derecho: la transformación de la cultura jurídica latinoamericana. Bogotá: Legis.

López-Medina, D. E. (2006a). El derecho de los jueces: obligatoriedad del precedente constitucional, análisis de sentencias y lineas jurisprudenciales y teoría del derecho judicial. Bogotá: Ediciones Uniandes; Legis Editores.

López-Medina, D. E. (2006b). Interpretación constitucional. Bogotá: Escuela Judicial Rodrigo Lara Bonilla.

Moncada, P. (2007). Los estados fallidos o fracasados: un debate inconcluso $y$ sospechoso. Bogotá: Siglo del Hombre Editores; Universidad de los Andes. 
Morales, L. G. (1988). El contrato de agencia mercantil: análisis comparativo entre derecho colombiano y el derecho anglosajón. Revista de Derecho Privado, 4, 39-69.

Namén, W. (1995). Derecho de la distribución comercial. Bogotá: El Navegante Editores.

Olano, H. A. (2010). Fuentes del Derecho: ley, costumbre, jurisprudencia, doctrina $y$ principios generales del derecho. Bogotá: Doctrina y Ley.

Ospina, E. y Ospina, G. (1998). Teoría General del contrato y del negocio jurídico. Bogotá: Temis.

Pájaro, N. (2009). El contrato y sus principios orientadores. En M. Castro de Cifuentes, Derecho de Obligaciones (Tomo 1, pp. 309-370). Bogotá: Temis.

Peña, L. (2010). Contratos mercantiles nacionales e internacionales. Bogotá: Editorial Temis; Universidad Santo Tomás.

Pérez, E. (2004). Derecho Internacional Privado. Madrid: Universidad Nacional de Educación a distancia.

Perilla, J. S. A. (2013). Alineación iusteórica desde las licencias creative commons. Revista de Derecho Privado, 50. doi: 10.15425/redepriv.50.2013.02

Perilla, J. S. A. (2015a). Alineación iusteórica de las fuentes del derecho comercial. Revista de Derecho Privado, 53. doi: 10.15425/ REDEpRIv.53.2015.03. Recuperado de https://derechoprivado.uniandes.edu.co/ components/com_revista/archivos/derechoprivado/pri481.pdf

Perilla, J. S. A. (2015b). Construcción antiformalista del consumidor medio. Revista de Derecho Privado, 54. doi: 10.15425/
REDEpRIv.54.2015.01 Recuperado de https://derechoprivado.uniandes.edu.co/ components/com_revista/archivos/derechoprivado/pri580.pdf

Perilla, J. S. A. (2015c). Contrato solemne para parejas del mismo sexo en Colombia. Revista de Derecho Público, 35. doi: 10.15425/redepub.35.2015.04

Perilla, J. S. A. (2016). El monismo impuro desde el Bloque de Constitucionalidad colombiano. En W. Alvez de Souza \& R. Rabinovich-Berkman (Comps.), Barajar $y$ dar de nuevo: trabajos sobre temas de derecho, de sociología y de historia jurídicas, dedicados a la Profesora Dra. Andrea Gastrón (pp. 395-411). Salvador, Bahía (Brasil): Editora Dois de Julho.

Pinzón, J. (1985). Introducción al Derecho Comercial. Bogotá: Temis.

Rodríguez, C. (1999). Una crítica contra los dogmas de la coherencia del derecho y la neutralidad de los jueces: los estudios críticos del derecho y la teoría de la decisión judicial. En D. Kennedy (Ed.), Libertad y restricción en la decisión judicial (pp. 17-88). Bogotá: Siglo del Hombre Editores.

Sanín, I. (1995). Derecho de la distribución comercial. Bogotá: El Navengante Editores.

Savigny, F (1979). Metodología Jurídica. Buenos Aires: Ediciones Depalma.

Sentencia del 15 de diciembre de 2006. Exp. 1992-09211-01. M. P. Fernando Giraldo Gutiérrez. Corte Suprema de Justicia, Sala de Casación Civil.

Sentencia del 4 de abril de 2008. Exp. 199800171-01. M. P. Ruth Marina Díaz Rueda. Corte Suprema de Justicia, Sala de Casación Civil. 
Sentencia del 24 de julio de 2012. Exp. 199821524-01. M.P. Fernando Giraldo Gutiérrez. Corte Suprema de Justicia, Sala de Casación Civil.

Sentencia del 10 de septiembre de 2013 (15 de julio anterior versión). Exp. 110013103022200500333-01. M. P. Fernando Giraldo Gutiérrez. Corte Suprema de Justicia, Sala de Casación Civil.

Sentencia C-901 (2011, noviembre, 30). Acción de inconstitucionalidad. M. P. Jorge Iván Palacio Palacio. Corte Constitucional.

Talero, S. (2010). La agencia comercial y los sistemas de distribución en el arbitraje internacional. Revista de Derecho Privado, 44. Recuperado de https://derechoprivado.uniandes.edu.co/components/com_revista/archivos/derechoprivado/pri71.pdf

Ureña, R. (2008). Derecho de las organizaciones internacionales. Bogotá D.C.: Editorial Temis.

Ureña, R. (2012). No citizens Here: Global Subjects and Participation in Internacional
Law. Estados Unidos: Martinus Nijhoff Publishers.

Ureña, R. (2015). Derecho Internacional: Poder y límites del derecho en la sociedad global. Bogotá D.C.: Ediciones Uniandes.

Valencia, A. (1985). Derecho Civil: De los contratos (Tomo 4). Bogotá: Editorial Temis.

Vallejo, F. (1999). El contrato de agencia mercantil. Bogotá: Editorial Legis.

Vernengo, R. J. (1977). La interpretación jurídica. Ciudad de México: Universidad Nacional Autónoma de México.

Wenzel, M. (1929). Juristische Grundprobleme - Zugleich eine Untersuchug zum Begriff des Staates und Problem des Völkerrechts. Berlín: F. Dümmlers.

Zuleta, E. (2004). Agencia comercial: ¿nueva tendencia jurisprudencial?. Foro de Derecho Mercantil, 2, 139-161. 